\title{
Optimality Condition of a Class of Arc Connected Function
}

\author{
Hailan Fu \\ Department of adult education \\ Tongren Polyrechnic, Tongren 100072, Guizhou, China \\ Xiaojun Lei \\ Department of Mathematics and Computer Science \\ Tongren Polyrechnic, Tongren 100072, Guizhou, China
}

\begin{abstract}
In the condition that the real valued function $f: S \rightarrow R$ is a arc connected function in arc connected set $S \subseteq R^{n}$, this paper give the definition of generalized arc connected function. The class function is the promotion of convex function which satisfies identified local-global extremum property. Conversely, under certain conditions. the function meeting local-global extremum property must be one of those generalized functions. Also, the optimality sufficient condition of $\min _{x \in S} f(x)$, s.t. $g(x) \leq 0$ is obtained under generalized connected assumption.
\end{abstract}

Keywords: Arc connected function, Generalized convexity, Local-global extremum property, Optimal condition

\section{Introduction}

In both theory and application, optimization has been developed very rapidly in recent 40 years. Optimization research has now become a very hot topic in academic theory and application. Along with depth study, we find many interesting results in theory, which make the object tense. From application point, functions involved in the problem are more close to reality, that is, more convenient to use. To make the research work more explicit, we introduce the optimization direction and area, which illustrates that the problem we study has nature, university and applicability.

The most important promotion of convexity is the inconvexity concept given by Hanson in 1981. After that, using generalized algebraic operation, Clarke generalized gradient, Minch symmetrical gradient and cone sub-differential concept, $(h, \phi) z$-(quasi)pseudo convexity, strict $(h, \phi) z-$ pseudo convexity, quasi Bs-inconvex, pseudo Bs-inconvexity, $(h, \phi) s-$ convexity, $(h, \phi) s$-(quasi)pseudo convexity, $(h, \phi) s$-(quasi)pseudo inconvexity, generalized uniform convexity, generalized uniform (quasi)pseudo convexity and $\eta-(A, N)$ function etc. 30 kinds of generalized convex functions were introduced, then the optimization research has become very in-depth and rich. Professor Hanson, in his paper, consider certain objective function from

$$
f(x)=f(a)+\nabla f(a)^{T}(x-a)+\cdots \cdots
$$

expanding in a point. For the proof that $f(x)$ takes extreme value in $x=a$, its factor $x-a$ can't afford a key role. Therefore he introduced the following inconvexity concept.

Definition 1.1 Suppose $f(x)$ be a differentiable function, $x \in R^{n}$ is its domain, $\alpha$ is one point in its domain. If existing a vector function $\eta: X \times X \rightarrow X$ satisfying

$$
f(x) \geq f(a)+\nabla f(a)^{T} \eta(x, a), \text { for all } \quad x \in X .
$$

then $f(x)$ is inconvex in point $a$. If $f(x)$ is inconvex in every point of $X$, then $f(x)$ is inconvex in $X$.

From (1), when point $a$ is settled, if the inner product of $\eta(x, a)$ and gradient of $f(x)$ in $a$ is greater than zero, where $\eta(x, a)$ is one of vector in $X$ defined by $a$ and $x$ for $x \in X$, then $f(x)$ can take local minimum in $a$.

In view of the above analysis, inconvex concept defined by Hanson brings the development and enrichment in optimization theory. One of the most prominent is the generation of weak convexity, characterization of optimal condition and the dual problem establishment. Validity characterization of multi-objective optimization problems and the dual problem of multiobjective optimization are more important. At the same time, the results in variational (multi-objective) problem and optimal control problem have similar results, which can be seen in all references(Zhian Liang, 2001- Weir T., 1998).

When $\eta(x, a)=x-a$, it is the usual convex concept. In this time. the gradient of $f(x)$ in point $a$ is an element in dual space of $X$, which can be seen as $\nabla f(a)$ acting on the element $x-a$ of $X$. In the inconvex set, $\nabla f(a)$ can be considered as the element in "dual space" acting on the set $\{\eta(x, a) \mid x \in X\}$. So from this point of view, it seems that we can also do some work, but it is not in the scope of this paper.

Let us consider the feasible region of optimization problem. Usually when we solve an optimization problem. Usually when we solve an optimization problem, the feasible region is generally a area with inner point. But in practical problems, 
it often does not have such conditions. For example, the feasible region is the following linear graph without inner point whose feasible region is connected by many curves(See Figure 1). Then the function defined in this feasible region can not be considered its derivation or directional derivation, and thus there is no way to consider gradient of this function.

For example, the region is a circle in $x y$-place and function is a curve in corresponding cylindrical surface, which is seen in Figure 2.

Considering this problem, Ortega and Rheinboldt(Ortega J.M., 1970) proposed arc connectivity concept of domain in 1970. They promoted the segment defined originally to a continuous arc. After that, Avriel and Zang(Avriel M., 1970) extended it to generalized convexity. Arc connectivity function and generalized function have good local-global extremum property. In this research area, also need to discuss the optimality condition and dual problems,also and the effective condition of multi-objective optimization and dual problems, which can be found in Minasian(Stancu Minasian I.M.) and references (Hansom M.A, 1980- Weir T., 1998). This paper introduces arc connectivity concept, define new concepts and give derivation results. Section 2 describes the basic concepts and basic conditions. Some properties of generalized arc connected function are given in section 3. Section 4 derives a main result.

\section{Basic Concepts and Basic Conclusions}

Definition 2.1 For arbitrary $x_{1}, x_{2} \in S$, if existing a continuous vector valued function $H_{x_{1}, x_{2}}:[0,1] \rightarrow S$ satisfying

$$
H_{x_{1}, x_{2}}(0)=x_{1}, H_{x_{1}, x_{2}}(1)=x_{2},
$$

then $S \subseteq R^{n}$ is arc connected, denoted by $\mathrm{AC}$ and $H_{x_{1}, x_{2}}$ is called arc.

Definition 2.2 Suppose $f(x)$ is a real valued function defined on a arc connected set $S \subseteq R^{n}$. For $x_{1}, x_{2} \in S, H_{x_{1}, x_{2}}$ is a arc connecting $x_{1}$ and $x_{2}$ in definition 2.1. For arbitrary $\lambda, 0 \leq \lambda \leq 1$, if

$$
f\left(H_{x_{1}, x_{2}}(\lambda)\right) \leq(1-\lambda) f\left(x_{1}\right)+\lambda f\left(x_{2}\right)
$$

holds, then $f(x)$ is called arc connected function in $S$.

Clearly, arc connectivity concept is the promotion of convexity. Noting that, if

$$
H_{x_{1}, x_{2}}(\lambda)=(1-\lambda) x_{1}+\lambda x_{2}
$$

arc connected set and arc connected function are equal to convex set and convex function respectively.

Paper (Zhian Liang) gives some examples of arc connected function. For example

$$
f(x)=100\left[x_{2}-\left(x_{1}\right)^{2}\right]^{2}+\left(1-x_{1}\right)^{2}
$$

is arc connected function on the arc

$$
H_{x^{1}, x^{2}}(\lambda)=\left[\begin{array}{c}
\lambda x_{1}^{2}+(1-\lambda) x_{1}^{1} \\
\left.\lambda\left[x_{2}^{2}-\left(x_{1}^{2}\right)^{2}\right]+(1-\lambda)\left[x_{2}^{1}-\left(x_{1}^{1}\right)^{2}\right]+\left[\lambda x_{1}^{2}+(1-\lambda) x_{1}^{1}\right]^{2}\right] .
\end{array}\right.
$$

This function $f(x)$ is called cut valley function.

Arc connected function still has good characters.

Theorem 2.1 (Avriel M., 1980) Suppose $f(x)$ is a arc connected function in a arc connected set $S \subseteq R^{n}$ and $x_{0}$ is a local minimum point of $f(x)$, then $x_{0}$ is a global minimum point of $f(x)$.

Now let us consider generalized arc connected function.

Definition 2.3 Suppose $f(x)$ is a real valued function in a arc connected set $S \subseteq R^{n}$ and $x_{0} \in S$. For arbitrary $x \in S$, if existing $H_{x, x_{0}}$ connected by $x$ and $x_{0}$ satisfying

$$
f(x) \leq f\left(x_{0}\right) \Rightarrow f\left(H_{x, x_{0}}(\lambda)\right) \leq f\left(x_{0}\right),
$$

for arbitrary $\lambda, 0 \leq \lambda \leq 1$, then $f(x)$ is a quasi-arc connected function, denoted by QACF; If $f(x) \leq f\left(x_{0}\right) \Rightarrow f\left(H_{x, x_{0}}(\lambda)\right)<$ $f\left(x_{0}\right)$ holds for $0 \leq \lambda \leq 1, f(x)$ is a strong quasi-arc connected function, denoted by SQACF; If $f(x)<f\left(x_{0}\right) \Rightarrow$ $f\left(H_{x, x_{0}}(\lambda)\right)<f\left(x_{0}\right)$ holds for $0 \leq \lambda \leq 1, f(x)$ is a strict quasi-arc connected function, denoted by STQACF; If $f(x)$ is QACF, SQACF and STQACF expectively in point of $S, f(x)$ is QACF, SQACF and STQACF expectively in $S$.

Contrasting (3) and (7), it is easy to see that the arc connected function is quasi-arc connected. At the same time, we have the following conclusions.

Theorem 2.2 Suppose $f(x)$ is a real valued function in a arc connected set $S \subseteq R^{n}, f(x)$ is QACF if and only if the level set

$$
S(f, \alpha)=\{x \in S, f(x) \leq \alpha\}
$$


is arc connected for $\alpha \in R$.

Proof: The necessity is obvious. Now we consider its sufficiency. Suppose $S(f, \alpha)$ is arc connected for $\alpha \in R$. Let $\alpha=\max \left\{f\left(x_{1}\right), f\left(x_{2}\right)\right\}$ for $x_{1}, x_{2} \in S$, then we can find arc $H_{x_{1}, x_{2}}$ connected by $x_{1}$ and $x_{2}$ in $S(f, \alpha)$ satisfying (2). For $f\left(x_{1}\right) \leq f\left(x_{2}\right)$ and $H_{x_{1}, x_{2}}$ is a vector valued function in $S(f, \alpha), f_{H_{x_{1}, x_{2}}} \leq f\left(x_{2}\right)$ for $\lambda$, where $0 \leq \lambda \leq 1$. And since $x_{1}$ and $x_{2}$ are arbitrary, then $f(x)$ is QACF.

Now, the following is an example of quasi-arc connected function.

Example 2.1 (Zhian Liang) Let $x \in R^{2}, f: R^{2} \rightarrow R$ is

$$
f(x)=\left\{\begin{array}{cc}
\left(x^{1} x^{2}\right)^{2}, & x^{1} x^{2} \leq 5 \\
25, & x^{1} x^{2}>5
\end{array}\right.
$$

$f(x)$ is QACF of the below arc

$$
H_{x_{1}, x_{2}}(\lambda)=\left\{\begin{array}{ll}
(1-2 \lambda) x_{1}, & 0 \leq \lambda \leq \frac{1}{2} \\
(2 \lambda-1) x_{2}, & \frac{1}{2} \leq \lambda \leq 1
\end{array} \text { for } x_{1}, x_{2} \in R^{2}\right.
$$

The figure of $f(x)$ is seen in Figure 3.

From definition, $\mathrm{STQACF} \Rightarrow \mathrm{SQACF} \Rightarrow \mathrm{QACF}$, but it does not hold in turn, which is referred in (Zhian Liang).

Define a function in a feasible arc connected domain. For example, objective function $f(x)$ in problem (ACP) may has good analysis property, or corner points appear. The following function $f(x)$ is an example(See figure 4).

Using analysis property to character that $f(x)$ takes extremum in a point in $S$, we should consider the directional derivative of function in this point, and then give the arc derivation concept.

Definition 2.4 Suppose $H_{x_{1}, x_{2}}$ is a arc connecting $x_{1}$ and $x_{2}$ in definition 2.1. If thee is a vector $\nabla^{-} H_{x_{1}, x_{2}}\left(\lambda_{0}\right) \in R^{n}$ and a vector function $\alpha:[0,1] \rightarrow R^{n}$ satisfying $\lim _{t \rightarrow 0} \alpha(t)=0$, and

$$
H_{x_{1}, x_{2}}(\lambda)=H_{x_{1}, x_{2}}\left(\lambda_{0}\right)+\left(\lambda-\lambda_{0}\right) \nabla^{-} H_{x_{1}, x_{2}}\left(\lambda_{0}\right)+\left(\lambda_{0}-\lambda\right) \alpha £\left(\lambda_{0}-\lambda\right)
$$

holds for $0 \leq \lambda \leq 1$, then vector $\nabla^{-} H_{x_{1}, x_{2}}\left(\lambda_{0}\right)$ is called directional derivative of $H_{x_{1}, x_{2}}$ in the point $\lambda=\lambda_{0}$, which is given by

$$
\nabla^{-} H_{x_{1}, x_{2}}\left(\lambda_{0}\right)=\lim _{\lambda \rightarrow \lambda_{0}}\left\{\left[H_{x_{1}, x_{2}}(\lambda)-H_{x_{1}, x_{2}}\left(\lambda_{0}\right)\right] /\left(\lambda-\lambda_{0}\right)\right\}
$$

Now, we define arc derivation concept of arc connected function.

Definition 2.5 Suppose $f(x)$ is a continuous real valued function in a arc connected set $S \subseteq R^{n}$ and $x_{0} \in S$. For $x \in S$, $H_{x, x_{0}}$ is a arc connecting $x$ and $x_{0}$ in definition 2.1. If $x$ approaches to $x_{0}$ along $H_{x, x_{0}}$ and the following limit exists:

$$
f_{H_{x, x_{0}}}\left(x_{0}\right)=\left(\nabla^{-} H_{x_{1}, x_{2}}(1)\right)^{T} \nabla f\left(x_{0}\right)=\lim _{\lambda \rightarrow 1} \frac{f\left(H_{x, x_{0}}(\lambda)\right)-f\left(x_{0}\right)}{\lambda-1},
$$

then $f(x)$ is arc derivative for $H_{x, x_{0}}$ in point $x_{0}$, which is denoted by $f_{H_{x, x_{0}}}\left(x_{0}\right)$.

For a continuous ACF in $S, f(x)$ can be expressed

$$
f\left(H_{x_{1}, x_{2}}(\lambda)\right)=f\left(x_{2}\right)+(\lambda-1) f_{H_{x_{1}, x_{2}}}+(1-\lambda) \bar{\alpha}(1-\lambda)
$$

for $0 \leq \lambda \leq 1$, where $\bar{\alpha}:[0,1] \rightarrow R$ satisfying $\lim _{t \rightarrow 0} \bar{\alpha}(t)=0$.

From arc derivation definition, we obtain a first-order necessary condition of $f(x)$ taking extremum.

Theorem 2.3 Suppose $f(x)$ is a real valued function in a arc connected set $S \subseteq R^{n}$ and $x_{0} \in S$. For $x \in S, H_{x, x_{0}}$ is a arc connecting $x$ and $x_{0}$ and $f(x)$ is arc derivative along $H_{x, x_{0}}$ in point $x_{0}$. If $f(x)$ takes maximum in this point, $f_{H_{x, x_{0}}}\left(x_{0}\right) \geq 0$; If $f(x)$ takes minimum in this point, $f_{H_{x, x_{0}}}\left(x_{0}\right) \leq 0$.

Now we define pseudo arc connectivity from arc derivation concept.

Definition 2.6 Suppose $f(x)$ is a real valued function in a $\operatorname{arc}$ connected set $S \subseteq R^{n}$ and $x_{0} \in S$. For $x \in S, H_{x, x_{0}}$ is a arc connecting $x$ and $x_{0}$, if

$$
f(x) \leq f\left(x_{0}\right) \Rightarrow f_{H_{x, x_{0}}}\left(x_{0}\right) \geq 0
$$

holds, then $f(x)$ is pseudo arc connected function, denoted by PACF. Under the same conditions, if $f(x) \leq f\left(x_{0}\right) \Rightarrow$ $f_{H_{x, x_{0}}}\left(x_{0}\right)>0$ holds, then $f(x)$ is strong pseudo arc connected function, denoted by SPACF; If $f(x)<f\left(x_{0}\right) \Rightarrow f_{H_{x, x_{0}}}\left(x_{0}\right)>$ 
0 holds, then $f(x)$ is strict pseudo arc connected function, denoted by STPACF; If $f(x)$ is PACF, SPACF and STPACF in arbitrary point of $S, f(x)$ is PACF, SPACF and STPACF in $S$.

From the definitions, the following holds:

I. $\mathrm{STPACF} \Rightarrow \mathrm{SPACF} \Rightarrow \mathrm{PACF}$;

II. $\mathrm{ACF} \Rightarrow \mathrm{STQACF} \Rightarrow \mathrm{SQACF} \Rightarrow \mathrm{QACF} \Rightarrow \mathrm{PACF}$.

\section{Local- Global Extremum Property of Generalized Arc Connected Function}

In the previous section, arc connected function has a good local-global extremum property. In this section, we discuss the necessary and sufficient condition for local-global extremum property of generalized arc connected function. First of all, that generalized arc connected function has determinate local-global extremum property is showed. Next, whether the function of determinate local-global extremum property belongs to those functions is discussed, which is regarded as the sufficient condition of local-global extremum property, and also regarded as the necessary condition of local-global extremum property. The part about QACF can be referred in (Zhian Liang, 2001) and (Zhian Liang).

Theorem 3.1 (Zhian Liang, 2001) Suppose $f(x)$ is QACF in a arc connected set $S \subseteq R^{n}$. If $x_{0} \in S$ is a strict local minimum point of $f(x)$, then $x_{0}$ is a strict global minimum point of $f(x)$ in $S$.

For SQACF, we have the following theorems.

Theorem 3.2 Suppose $f(x)$ is SQACF in a arc connected set $S \subseteq R^{n}$. If $x_{0} \in S$ is a local minimum point of $f(x)$, then $x_{0}$ is the only global minimum point of $f(x)$ in $S$.

Proof: We use disproof method. Suppose $f(x)$ is SQACF and $x_{0} \in S$ is a local minimum point of $f(x)$. If there is $\bar{x} \in S$ satisfying $f(\bar{x})<f\left(x_{0}\right)$, then it exists arc $H_{\bar{x}, x_{0}}$ connecting $\bar{x}$ and $x_{0}$ satisfying

$$
f\left(H_{\bar{x}, x_{0}}(\lambda)\right)<f\left(x_{0}\right) \text { for } 0 \leq \lambda<1
$$

For arbitrary neighborhood of $x_{0}$, we can always find $\lambda_{0}$. When $\lambda_{0} \leq \lambda \leq 1, H_{\bar{x}, x_{0}}$ is in this neighborhood, which is a contradiction that $x_{0} \in S$ is a local minimum point of $f(x)$. Theorem is proven.

For STQACF, we have the following theorems.

Theorem 3.3 Suppose $f(x)$ is STQACF in a arc connected set $S \subseteq R^{n}$. If $x_{0} \in S$ is a local minimum point of $f(x)$, then $x_{0}$ is a global minimum point of $f(x)$ in $S$.

For PACF defined by us, we have the following properties:

Theorem 3.4 Suppose $f(x)$ is a real valued function in a arc connected set $S \subseteq R^{n}$ and $x_{0} \in S$ satisfies that $\nabla f\left(x_{0}\right)=0$. If $f(x)$ is STQPACF, $x_{0}$ is a global minimum point of $f(x)$ in $S$; If $f(x)$ is SPACF, $x_{0}$ is the only global minimum point of $f(x)$ in $S$.

Proof: Suppose $f(x)$ is STPACF and $x_{0} \in S$ satisfing that $\nabla f\left(x_{0}\right)=0$. For arbitrary $x \in S$ and the corresponding arc $H_{x, x_{0}}$,

$$
f_{H_{x, x_{0}}}\left(x_{0}\right)=\left(\nabla^{-} H_{x, x_{0}}(1)\right) \nabla f\left(x_{0}\right)=0
$$

holds. From the definition of STPACF, $f(x) \geq f\left(x_{0}\right)$ holds, i.e. $x_{0}$ is a global minimum point of $f(x)$ in $S$. If $f(x)$ is SPACF and from its definition, $f(x)>f\left(x_{0}\right)$ ) holds for $x \in S, x \neq x_{0}$, i.e. $x_{0}$ is the only global minimum point of $f(x)$ in $S$. Theorem is proven.

Before discussing the sufficiency of local-global property, we first define that

$$
S^{\circ}(f, \alpha)=\{x \in S: f(x)<\alpha\}
$$

is a strict level set of $f(x)$ in point $\alpha \in R$. Now a relevant lemma is shown.

Lemma 3.1 Suppose $f(x)$ is QACF in a arc connected set $S \subseteq R^{n}$, then $S^{\circ}(f, \alpha)$ is arc connected for any $\alpha \in R$.

(By this lemma, we can get the following results).

Theorem 3.5 Suppose $f(x)$ is QACF in a arc connected set $S \subseteq R^{n}$ and each local minimum point of $f(x)$ is also global minimum point, then $f(x)$ is STQACF.

Proof: Suppose $f\left(x_{1}\right)<f\left(x_{2}\right)$ for any $x_{1}, x_{2} \in S$. Let $\alpha=f\left(x_{2}\right)$, then $x_{1} \in S^{\circ}(f, \alpha)$. Since $f(x)$ is QACF and from lemma $3.1, S^{\circ}(f, \alpha)$ is arc connected. From the definition of arc connected set, arc $H_{x_{1}, x_{2}}$ exists in $S^{\circ}(f, \alpha)$ satisfying

$$
H_{x_{1}, x_{2}}(0)=x_{1}, H_{x_{1}, x_{2}}(1)=x_{2},
$$

and $f\left(H_{x_{1}, x_{2}}(\lambda)<f\left(x_{2}\right)\right.$ holds for $0 \leq \lambda<1$, i.e. $f(x)$ is STQACF. Theorem is proven. 
Through $S^{\circ}(f, \alpha)$, we can also describe QACF as follows.

Theorem 3.6 Suppose $f(x)$ is a real valued function in a arc connected set $S \subseteq R^{n}$. If $S^{\circ}(f, \alpha)$ is arc connected for $\alpha \in R$ and each local minimum point of $f(x)$ is also global minimum point, then $f(x)$ is STQACF.

Theorem 3.7 Suppose $f(x)$ is QACF in a arc connected set $S \subseteq R^{n}$. If the only local minimum of $f(x)$ is also global minimum, then $f(x)$ is SQACF.

Proof: Suppose $f(x)$ satisfies the condition in theorem 3.7. From theorem 3.5, $f(x)$ is STQACF, i.e. $f\left(x_{1}\right)<f\left(x_{2}\right) \Rightarrow$ $f\left(H_{x_{1}, x_{2}}(\lambda)\right)<f\left(x_{2}\right)$ for $x_{1}, x_{2} \in S$ and $0 \leq \lambda<1$. So we only consider $f\left(x_{1}\right)=f\left(x_{2}\right)$ for proving that $f(x)$ is SQACF.

Suppose $f\left(x_{1}\right)=f\left(x_{2}\right)=\alpha$ and $x_{1} \neq x_{2}$. Obviously, $x_{1}$ and $x_{2}$ are not the global minimum point of $f(x)$ and $S^{\circ}(f, \alpha)$ is nonempty. Take $\bar{x} \in S^{\circ}(f, \alpha)$ and since $f(x)$ is STQACF, then existing arc $H_{\bar{x}, x_{1}}$ and $H_{\bar{x}, x_{2}}$ satisfying

$$
f\left(H_{\bar{x}, x_{1}}(\lambda)\right)<f\left(x_{1}\right), f\left(H_{\bar{x}, x_{2}}(\lambda)\right)<f\left(x_{2}\right) .
$$

for $0 \leq \lambda<1$. Let

$$
H_{x_{1} x_{2}}(\lambda)=\left\{\begin{array}{ll}
H_{\bar{x}, x_{1}}(1-2 \lambda), & 0 \leq \lambda \leq \frac{1}{2} \\
H_{\bar{x}, x_{2}}(2 \lambda-1), & \frac{1}{2} \leq \lambda \leq 1
\end{array},\right.
$$

then

$$
f\left(H_{x_{1}, x_{2}}(\lambda)\right)<f\left(x_{2}\right)
$$

for $0<\lambda<1$. i.e. $f(x)$ is SQACF. Theorem is proven.

Similarly, we can prove the following generalized conclusions.

Theorem 3.8 Suppose $f(x)$ is a real valued function in a $\operatorname{arc}$ connected set $S \subseteq R^{n}$. If $S^{\circ}(f, \alpha)$ is arc connected for $\alpha \in R$ and the only local minimum point of $f(x)$ is also global minimum point, then $f(x)$ is SQACF.

Now, we discuss what conditions PACF satisfies.

Theorem 3.9 Suppose $f(x)$ is arc derivative QACF in a arc connected set $S \subseteq R^{n}$. If the only stable point of $f(x)$ is also the global minimum point, then $f(x)$ is SPACF.

Proof: Suppose $f(x)$ is arc derivative QACF in a arc connected set $S \subseteq R^{n}$. For $x_{1}, x_{2} \in S$,

1) $f\left(x_{1}\right)<f\left(x_{2}\right) . \nabla f\left(x_{2}\right) \neq 0$ is known. From the continuity of $f(x)$, it exists $0<\lambda_{0}<1$ making

$$
\underline{f\left(x_{1}\right)<f\left[x_{2}-(1-\lambda) \nabla f\left(x_{2}\right)\right]<f\left(x_{2}\right)}
$$

for all $\lambda_{0} \leq \lambda \leq 1$. Let

$$
\underline{\bar{x}=x_{2}-\left(1-\lambda_{0}\right) \nabla f\left(x_{2}\right)} .
$$

Since $f\left(x_{1}\right)<f(\bar{x})$, there is a $\operatorname{arc} H_{x_{1}, \bar{x}}(\lambda)$ making

$$
f\left(H_{x_{1}, \bar{x}}(\lambda)\right) \leq f(\bar{x})
$$

for $0 \leq \lambda<1$. Now we define

$$
H_{x_{1}, x_{2}}(\lambda)=\left\{\begin{array}{cc}
H_{x_{1}, \bar{x}}\left(\frac{\lambda}{\lambda_{0}}\right), & 0 \leq \lambda \leq \lambda_{0} \\
x_{2}-(1-\lambda) \nabla f\left(x_{2}\right), & \lambda_{0} \leq \lambda \leq 1
\end{array}\right.
$$

then

$$
\begin{gathered}
\nabla^{-} H_{x_{1}, x_{2}}(1)=\nabla f\left(x_{2}\right), \\
f_{(} H_{x_{1}, x_{2}}\left(x_{2}\right)=\left(\nabla^{-} H_{x_{1}, x_{2}}(1)\right)^{T} \nabla f\left(x_{2}\right)=\left\|\nabla f\left(x_{2}\right)\right\|^{2}>0 .
\end{gathered}
$$

1) $f\left(x_{1}\right)=f\left(x_{2}\right)$. From hypothesis, $\nabla f\left(x_{1}\right) \neq 0$ and $\nabla f\left(x_{2}\right) \neq 0$. Similarly with 1$)$, we can find $0<\lambda_{1}<1,0<\lambda_{2}<$ $1, \lambda_{1}<\lambda_{2}$ making

$$
\begin{gathered}
f\left(x_{1}-\lambda \nabla f\left(x_{1}\right)\right)<f\left(x_{1}\right) \quad \text { for } \quad 0<\lambda \leq \lambda_{1}, \\
f\left(x_{2}-(1-\lambda) \nabla f\left(x_{2}\right)\right)<f\left(x_{2}\right) \quad \text { for } \quad \lambda_{2} \leq \lambda<1 .
\end{gathered}
$$

Let

$$
\overline{x_{1}}=x_{1}-\lambda_{1} \nabla f\left(x_{1}\right), \overline{x_{2}}=x_{2}-(1-\lambda) \nabla f\left(x_{2}\right) .
$$

From theorem 3.7, $f(x)$ is SQACF. So it exists arc $H_{\overline{x_{1}}, \overline{x_{2}}}$ making

$$
f\left(H_{\overline{x_{1}}, \overline{x_{2}}}(\lambda)\right)<\max \left\{f\left(\overline{x_{1}}\right), f\left(\overline{x_{2}}\right)\right\}<f\left(x_{2}\right)=f\left(x_{1}\right)
$$


for $0<\lambda<1$. Let

$$
H_{x_{1}, x_{2}}(\lambda)=\left\{\begin{array}{cc}
x_{1}-\lambda \nabla f\left(x_{1}\right), & 0 \leq \lambda \leq \lambda_{1}, \\
H_{\overline{x_{1}}, \overline{x_{2}}}\left(\frac{\lambda-\lambda_{1}}{\lambda_{2}-\lambda_{1}}\right), & \lambda_{1} \leq \lambda \leq \lambda_{2}, \\
x_{2}-(1-\lambda) \nabla f\left(x_{2}\right), & \lambda_{2} \leq \lambda \leq 1,
\end{array}\right.
$$

then

$$
\begin{gathered}
\nabla^{-} H_{x_{1}, x_{2}}(1)=\nabla f\left(x_{2}\right), \\
f_{H_{x_{1}, x_{2}}}\left(x_{2}\right)=\left(\nabla^{-} H_{x_{1}, x_{2}}(1)\right)^{T} \nabla f\left(x_{2}\right)=\left\|\nabla f\left(x_{2}\right)\right\|^{2}>0 .
\end{gathered}
$$

Summing up 1) and 2) and from the definition of SPACF, $f(x)$ is SPACF. Theorem is proven.

Theorem 3.10 Suppose $f(x)$ is a continuous real valued function in a arc connected set $S \subseteq R^{n}$ and $S^{\circ}(f, \alpha)$ is arc connected. If the only stable point of $f(x)$ is also the global minimum point, then $f(x)$ is SPACF, and also is PACF.

Directly from theorem 3.9, the following conclusion is gotten.

Theorem 3.11 Suppose $f(x)$ is arc derivative QACF in a arc connected set $S \subseteq R^{n}$. If every stable point of $f(x)$ is also the global minimum point, then $f(x)$ is STPACF, and also is PACF.

Theorem 3.12 Suppose $f(x)$ is a continuous real valued function in a arc connected set $S \subseteq R^{n}$ and $S^{\circ}(f, \alpha)$ is arc connected. If every stable point of $f(x)$ is also the global minimum point, then $f(x)$ is STPACF, and also is PACF.

\section{Optimization condition of Generalized Arc Connected Function and Dual Theory}

In this section, we consider the following optimal problem:

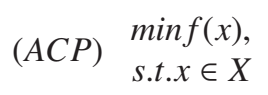

where $X=\left\{x \in S: g_{j}(x) \leq 0, j=1,2, \cdots, m\right\}, f(x)$ and $g_{j}(x), j=1,2, \cdots, m$ is a real valued function in a arc connected set $S \subseteq R^{n}$, and $f(x)$ and $g_{j}(x), j=1,2, \cdots, m$ is arc connected about $H_{x_{1}, x_{2}}$ for $x_{1}, x_{2} \in S$ and arc $H_{x_{1}, x_{2}}$ is connected by $x_{1}$ and $x_{2}$.

First, the sufficiency is shown.

Theorem 4.1 Suppose $X=\left\{x \in S: g_{j}(x) \leq 0, j=1,2, \cdots, m\right\}$ is a feasible domain in problem (ACP) and $f(x)$ and $g_{j}(x), j=1,2, \cdots, m$ is arc connected in the arc connected set $S \subseteq R^{n}$. If $x^{*}$ is the optimal solution of (ACP) and $f_{H_{x, x^{*}}}\left(x^{*}\right)$ and $\left(g_{I}\right)_{H_{x, x^{*}}}\left(x^{*}\right)$ are convex function for $x$, then it exists $r_{0}^{*} \in R, r^{*} \in R^{m}$ satisfying

$$
\begin{gathered}
r_{0}^{*} f_{H_{x, x^{*}}}\left(x^{*}\right)+r^{* T}\left(g_{I}\right)_{H_{x, x^{*}}}\left(x^{*}\right) \leq 0, \\
r^{* T} g\left(x^{*}\right)=0, \\
\left(r_{0}^{*}, r^{*}\right) \geq 0
\end{gathered}
$$

for $x \in S$, where $I:=I\left(x^{*}\right):=\left\{i \mid g_{i}\left(x^{*}\right)=0\right\}, J:=J\left(x^{*}\right):=\left\{j \mid g_{j}\left(x^{*}\right)<0\right\}$.

Proof: First we prove equation system

$$
\begin{gathered}
f_{H_{x, x^{*}}}\left(x^{*}\right)>0, \\
\left(g_{I}\right)_{H_{x, x^{*}}}\left(x^{*}\right)>0
\end{gathered}
$$

has no solutions in $S$.

We use disproof method. Suppose $x \in S$ is a solution of equation system (35) and (36). Since $f_{H_{x, x^{*}}}\left(x^{*}\right)$ and $\left(g_{I}\right)_{H_{x, x^{*}}}\left(x^{*}\right)$ exist, then for $0 \leq \lambda \leq 1$,

$$
\begin{gathered}
f\left(H_{x, x^{*}}(\lambda)\right)=f\left(x^{*}\right)+(\lambda-1) f_{H_{x, x^{*}}}\left(x^{*}\right)+(1-\lambda) \alpha(1-\lambda), \\
g_{i}\left(H_{x, x^{*}}(\lambda)\right)=g_{i}\left(x^{*}\right)+(\lambda-1)\left(g_{i}\right)_{H_{x, x^{*}}}\left(x^{*}\right)+(1-\lambda) \alpha_{i}(1-\lambda),
\end{gathered}
$$

where

$$
\begin{gathered}
\alpha:[0,1] \rightarrow R, \quad \lim _{t \rightarrow 0} \alpha(t)=0, \\
\alpha_{i}:[0,1] \rightarrow R, \quad \lim _{t \rightarrow 0} \alpha(t)=0, i \in I
\end{gathered}
$$

From (35),(36),(37) and (38), we can obtain that

$$
\begin{aligned}
& f_{H_{x, x^{*}}}\left(x^{*}\right)-\alpha(1-\lambda)>0 ; \\
& \left(g_{i}\right)_{H_{x, x^{*}}}\left(x^{*}\right)-\alpha_{i}(1-\lambda)>0, i \in I
\end{aligned}
$$


for enough $\lambda$, denoted by $\lambda_{0}<\lambda<1$. Then from (35) and (36), for $\lambda_{0}<\lambda<1$,

$$
\begin{gathered}
f\left(H_{x, x^{*}}(\lambda)\right)-f\left(x^{*}\right)<0, \\
\left(g_{i}\right) H_{x, x^{*}}(\lambda)-g_{i}\left(x^{*}\right)<0, i \in I .
\end{gathered}
$$

Since $g_{i}, j \in J$ is arc derivative in $x^{*}$, so it is continuous. Simultaneously£ $\operatorname{arc} H_{x, x^{*}}(\lambda)$ is a continuous function about $\lambda$. So

$$
\lim _{\lambda \rightarrow 1} g_{j}\left(H_{x, x^{*}}(\lambda)\right)=g_{j}\left(x^{*}\right)<0 .
$$

This implies that it exists $\lambda_{j}^{*}, j \in J$ making

$$
g_{i}\left(H_{x, x^{*}}(\lambda)\right)<0
$$

when $\lambda_{j}^{*} \leq \lambda \leq 1$. Let $\lambda^{*}=\max \left\{\lambda_{0}, \lambda_{j}^{*}\right\}$, from (41), (42) and (43), we obtain that $H_{x, x^{*}}(\lambda) \in X$ for $\lambda^{*}<\lambda<1$ and $f\left(H_{x, x^{*}}(\lambda)\right)-f\left(x^{*}\right)<0$, which conflicts with that $x^{*}$ is the optimal solution of (ACP). Therefore equation system (35) and (36) has no solutions.

Because $f_{H_{x, x^{*}}}\left(x^{*}\right)$ and $\left(g_{I}\right)_{H_{x, x^{*}}}\left(x^{*}\right)$ are convex functions about $x$, there are incomplete zero $r_{0}^{*} \in R$ and $r_{i}^{*} \in R^{m}$ satisfying

$$
r_{0}^{*} f_{H_{x, x^{*}}}\left(x^{*}\right)+r_{I}^{* T}\left(g_{I}\right)_{H_{x, x^{*}}}\left(x^{*}\right) \leq 0
$$

for $x \in S$. Let $r_{J}^{*}=0$, the theorem is proven.

\section{References}

Antczak T. (2001). On (p,r)łinvexityłtypes Nonlinear Programming Problems. J Math Anal Appl., 264:382-397.

Antczak T. (2001). (p,r)łinver sets and functions. J Math Anal Appl., 263:355-379.

AVRIEL M. (1976). Nonlinear Programming: Analysis and Method. New Jersey: PrenticełHall, Cliffs

Avriel M., Zang, I. (1980). Generalized arcwise connected sets and functions and characterization of local-global minimum properties, Journal of optimization Theory and Applications, 32, 407-425.

Hanson M A. (1980). On sufficienecy of the Kuhn--Tuck conditions. J Math Anal Appl., 80:545-550.

Ortega J.M. and Rheinboldt W.C. (1970). Iterative solutions of noonliear equations in several variables, Acadamic Press, New York.

Stancu Minasian I.M. Cabalero R., and Muaoz M.M., Duality in fractional programming involving locally arewise connected sets and related functions, (to appear).

Weir T, Mond B. (1998). Preinvex Functions in Multiple Objective Optimization. J Math Anal Appl., 136:29-38.

Zhian Liang, Hong Xuan Huang, P. M. Pardalos. (2001). Optimality conditions for a class of fractional programmings, Journal of Optimization Theory and Applications, 110 (3).

Zhian Liang, Hong Xuan Huang, P. M. Pardalos. Efficiency conditions and duality for a class of multiobjective fractional programming problems, Journal of Global Optimization, 27 (2).

Zhian Liang, Qinkai Ye. (1999). Proper Efficiency Conditions for a Class of Multiobjective Fractional Optimal Control Problems. Journal of Peking University, 35(5).

Zhian Liang, Qinkai Ye. (2001). Duality for a class of multiobjective control problems with generalized invexity. J. Math. Anal. Appl., 256(2).

Zhian Liang, Qinkai Ye. (1999). The General Duality for a Class of Multiobjective Variational Problems with Generalized Invexity, Journal of Inner Mongolia University, 30 (1).

Zhian Liang, Zhen-wei Shi. (2003). Optimality conditions and duality for a minimax fractional programming with generalized convexity, J. Math. Anal. Appl., 277.

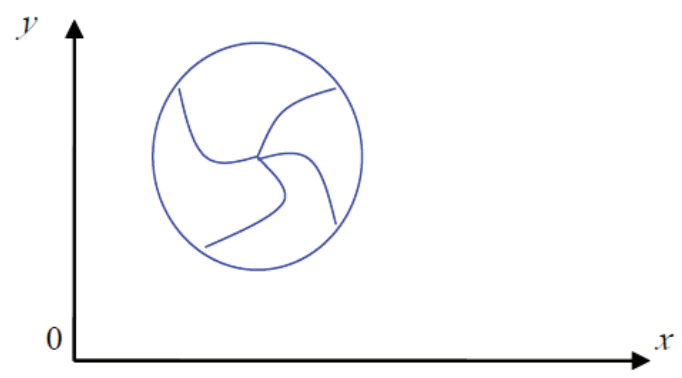

Figure 1. 


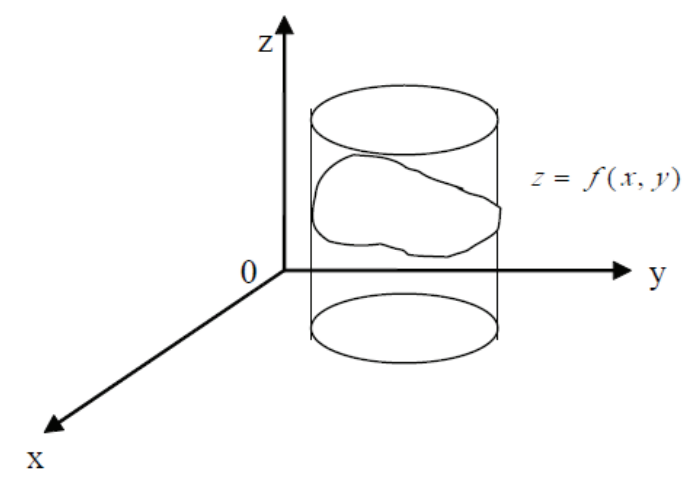

Figure 2.

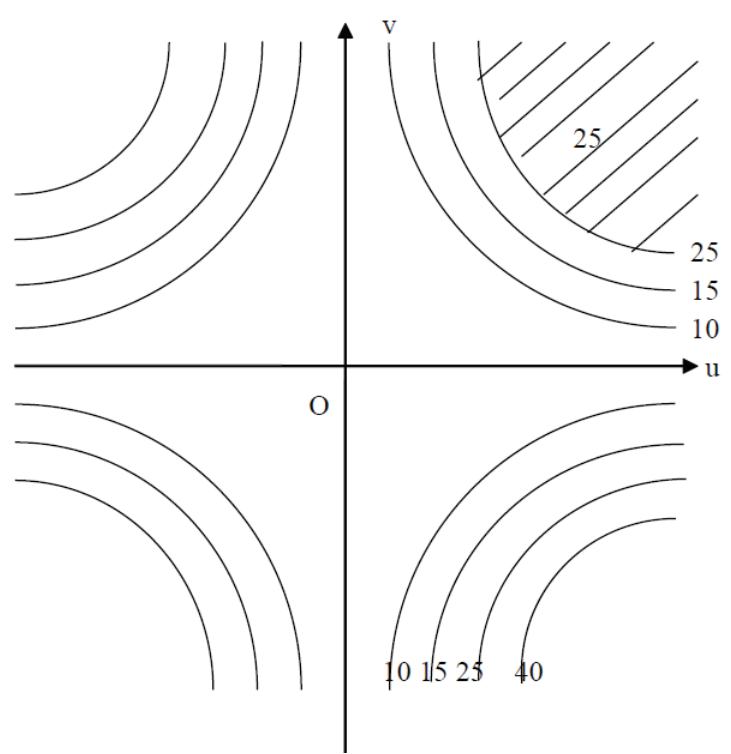

Figure 3.

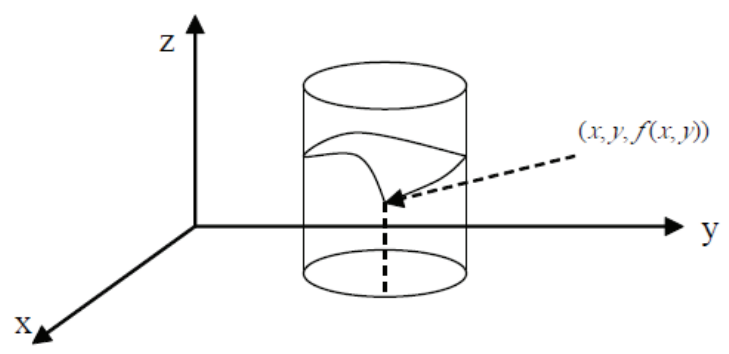

Figure 4. 